\title{
ESTRATÉGIAS PARA CONSOLIDAÇÃO DA COORDENAÇÃO DO CUIDADO PELA ATENÇÃO BÁSICA
}

\author{
STRATEGIES FOR THE CONSOLIDATION OF THE COORDINATION OF CARE BY PRIMARY CARE
}

\author{
ESTRATEGIAS PARA CONSOLIDACIÓN DE LA COORDINACIÓN DEL CUIDADO POR PARTE \\ DE LA ATENCIÓN BÁSICA
}

\author{
Patty Fidelis de Almeida ${ }^{1}$ \\ Juliana Marin $^{2}$ \\ Elisete Casotti ${ }^{3}$
}

Resumo $\mathrm{O}$ estudo aqui apresentado analisou a coordenação do cuidado por meio de dados do Programa Nacional para a Melhoria da Qualidade e do Acesso da Atenção Básica. Tratou-se de estudo descritivo com base em questionários aplicados a 1.313 usuários e 324 equipes de atenção básica no município do Rio de Janeiro em 2012. Avaliaram-se dimensões como organização da porta de entrada, resolutividade e continuidade do cuidado, integração horizontal, organização dos fluxos e acesso à rede de referência, continuidade informacional e comunicação entre profissionais. Os resultados indicaram que a atenção primária em saúde se consolidou como porta de entrada preferencial. Os usuários relataram que as equipes de atenção básica buscavam resolver seus problemas de saúde, o prontuário eletrônico estava disponível, embora não fosse integrado aos demais níveis, e os profissionais indicaram realizar reuniões semanais e receber apoio matricial. Entretanto, o tempo de espera para atendimento especializado era alto e a comunicação entre os profissionais insuficiente, o que dificultava o percurso do usuário na busca pelo cuidado e desvelava as fragilidades do trabalho em rede. Foram identificados avanços no fortalecimento da atenção primária e desafios para a constituição da Rede de Atenção à Saúde que minimizavam as possibilidades de coordenação do cuidado pelas equipes de atenção básica.

Palavras-chave avaliação de serviços de saúde; atenção primária à saúde; estratégia saúde da família; coordenação do cuidado.
Abstract This study examined the coordination of care based on data from the National Program for the Improvement of Quality and Access to Primary Care. It was a descriptive study based on questionnaires answered by 1,313 users and 324 primary care teams in the city of Rio de Janeiro (Brazil), in 2012. Dimensions such as the organization of the gateway, resolution and continuity of care, horizontal integration, organization of flows and access to the referral network, informational continuity, and communication among professionals were analyzed. The results indicated that primary health care has established itself as the preferred gateway. Users reported that primary care teams sought to solve their health issues, the electronic medical record was available, although it had not been integrated into the other levels, and the professionals stated they held weekly meetings and got matrix support. However, there was a long waiting time for specialized care and a lack of communication among professionals, a fact that not only hindered the users in their pursuit for care, but also revealed networking weaknesses. There was progress in the process of strengthening primary care and challenges to the establishment of the Health Care Network that minimized the possibilities for care coordination by the primary care teams.

Keywords health service evaluation; primary health care; family health strategy; coordination of care. 


\section{Introdução}

A coordenação do cuidado é condição necessária para alcance de uma resposta integral, capaz de atender ao conjunto das necessidades de saúde dos usuários. A literatura internacional converge ao apontar que a fragmentação do cuidado é uma das principais causas da má qualidade da atenção, associada a custos mais elevados, duplicação e sobreutilização de procedimentos diagnósticos, uso de múltiplos medicamentos e planos terapêuticos conflitantes, sendo os efeitos negativos mais potentes sobre as condições crônicas (Nolte e McKee, 2008; Bodenheimer, 2008; Kringos et al., 2010; Bynum e Ross, 2012; Schang, Waibel e Thomson, 2013).

Schang, Waibel e Thomson (2013) elencam elementos organizacionais necessários para assegurar a coordenação do cuidado, como definição de metas compartilhadas para o sistema de saúde (níveis primário, secundário e terciário); esquemas de pagamento e alocação de recursos como incentivo para a coordenação; mecanismos de comunicação entre profissionais; desenvolvimento de uma cultura comum e de liderança orientadas ao trabalho em equipe; e fortalecimento da atenção primária à saúde (APS) como base do sistema de saúde. Embora existam inovações que possam contribuir para a melhor coordenação, maior ênfase deve ser dada à forma como o sistema de saúde é organizado em sua totalidade. Nesse sentido, o fortalecimento da APS seria a mais eficiente macropolítica para promover a coordenação do cuidado (Bodenheimer, 2008).

O debate na literatura internacional sustenta a importância da coordenação do cuidado, fortemente apoiada por sistemas que facilitam a comunicação entre prestadores. Segundo Bodenheimer (2008), para que a APS seja o centro da atenção, todas as informações devem estar disponíveis para seus profissionais e usuários. Múltiplos provedores independentes, sem um eixo articulador, não conseguem definir claramente suas responsabilidades.

Nos sistemas de saúde, intervenções pró-coordenação podem ser desenvolvidas nos níveis macro (mecanismos regulatórios e esquemas de pagamento), meso (criação de funções gerenciais compartilhadas como comitês de gestão) e micro (introdução de sistema integrado de informações gerenciais, clínicas e administrativas a ser desenvolvido e implementado por gestores e profissionais de saúde) (Vázquez et al., 2005).

Bodenheimer (2008) destaca que a coordenação não envolve somente a integração entre múltiplos provedores e profissionais (APS e especialistas, serviços de emergência, procedimentos diagnósticos e hospital), mas inclui os usuários e seus familiares. A Agency for Health Care Research and Quality, segundo McDonald e colaboradores (2011), com base em mais de quarenta definições de coordenação encontradas na literatura, também propõe um 
conceito que incorpora o usuário como corresponsável pela organização de suas ações de cuidado.

McDonald e colaboradores (2011) reconhecem que coordenação significa diferentes coisas para diferentes atores e que uma definição consensual não foi amplamente desenvolvida. No estudo apresentado neste artigo, o atributo da coordenação do cuidado é compreendido como a articulação entre diversos serviços, ações e profissionais na atenção à saúde, de forma que, independentemente do local onde seja prestada, esteja sincronizada e voltada ao alcance de um objetivo comum (Vázquez et al., 2005; Almeida et al., 2010). Sustenta-se na existência de ações integradas entre prestadores e profissionais, conformados em rede, guiadas pela APS (Boerma, 2010).

No Brasil, a partir de 2004, iniciativas federais começaram a sinalizar a importância de se monitorarem a qualidade e a capacidade resolutiva da expansão da Estratégia Saúde da Família (ESF). Pesquisas de avaliação e monitoramento com foco nos atributos da atenção básica (AB) e propostas de autoavaliação da qualidade (Brasil, 2005) são alguns exemplos. O Programa Nacional de Melhoria do Acesso e da Qualidade da Atenção Básica (PMAQ-AB), cujo primeiro ciclo teve início em 2012, faz parte das recentes iniciativas do Ministério da Saúde para produzir mudanças nas condições de infraestrutura e funcionamento das unidades básicas de saúde (UBSs), qualificar o acesso e o processo de trabalho na atenção básica por meio de processos contínuos de avaliação e incentivos financeiros (Brasil, 2012a).

Fausto e colaboradores (2014), em estudo dos resultados do PMAQ-AB, reafirmam que a coordenação do cuidado pela atenção básica é dependente da integração entre serviços de saúde de diferentes densidades tecnológicas e facilitada por meio de fluxos assistenciais, continuidade informacional, porta de entrada pela $\mathrm{AB}$ com acesso oportuno e resolutivo, estratégias de acolhimento, atendimento à demanda espontânea, oferta abrangente de ações e de apoio matricial às equipes de atenção básica (EqABs).

O presente trabalho adotou a premissa de que são as EqABs as mais aptas a assumirem a função de coordenar o percurso terapêutico dos usuários no interior da Rede de Atenção à Saúde. Entretanto, ratifica-se que somente uma APS ou atenção básica, conforme nomenclatura adotada na Política Nacional de Atenção Básica (Brasil, 2012b), fortalecida em seus atributos essenciais, capaz de mobilizar apoios, recursos políticos, econômicos, financeiros e humanos, pode ser responsável pela coordenação do cuidado (Haggerty, Yavich e Báscolo, 2009).

Com base nos resultados do componente de avaliação externa do PMAQ-AB, o estudo aqui apresentado teve como objetivo analisar variáveis que compõem duas importantes estratégias associadas à consolidação da coordenação do cuidado: de fortalecimento da $\mathrm{AB}$ e de integração da Rede de Atenção à Saúde. 


\section{Desenho do estudo}

Tratou-se de um estudo descritivo, circunscrito às equipes que atuavam na Estratégia Saúde da Família (EqAB) 4 na cidade do Rio de Janeiro, cuja participação no ciclo I do PMAQ-AB foi homologada.

O desenho operacional do PMAQ-AB compreendeu quatro fases complementares: adesão e contratualização; desenvolvimento; avaliação externa; e pactuação. A etapa do desenvolvimento envolveu as equipes e os gestores em processos de autoavaliação, monitoramento, educação permanente e apoio institucional. Já a avaliação externa buscou reconhecer e valorizar os esforços e resultados das EqABs e dos gestores municipais na qualificação da atenção básica (Brasil, 2012a). Essa etapa foi executada por instituições de ensino e pesquisa parceiras do Ministério da Saúde, que realizaram visitas a cada uma das equipes que aderiram ao PMAQ-AB. Questionários estruturados sobre infraestrutura, insumos, organização do processo de trabalho e percepção dos usuários sobre o funcionamento do serviço foram aplicados in loco por entrevistadores. No município, os dados foram coletados de junho a agosto de 2012.

Na pesquisa que deu origem a este artigo, foram utilizadas variáveis do instrumento de avaliação externa do Saúde mais perto de você: acesso e qualidade do 'Módulo II - Entrevista com profissional da equipe de atenção básica e verificação de documentos na unidade de saúde', respondido por 323 equipes de saúde da família e uma EqAB parametrizada, e do ‘Módulo III - Entrevista na unidade de saúde com usuário', respondido por 1.313 usuários. Os respondentes do Módulo II foram um profissional de nível superior indicado pela EqAB e quatro usuários por equipe para o Módulo III selecionados pelos entrevistadores no dia da coleta. A seleção dos usuários atendeu aos critérios de maioridade, vínculo com uma EqAB e realização de pelo menos um atendimento na UBS nos últimos 12 meses.

Como a inclusão das EqABs atendeu ao critério de adesão e homologação das equipes ao programa, que no ciclo I se restringiu até $50 \%$ das equipes completas existentes em cada município, o banco não se constituiu em uma amostra representativa do conjunto das EqABs da cidade do Rio de Janeiro, mas exclusivamente ao universo das participantes do PMAQ-AB que, no ciclo I, provavelmente, representavam aquelas mais bem estruturadas. Outra limitação referiu-se à natureza da avaliação, que por estar associada a um processo de certificação, com repasse financeiro por performance, estava suscetível às pressões por desempenho.

O estudo foi submetido ao Comitê de Ética em Pesquisa em Seres Humanos da Escola Nacional de Saúde Pública Sergio Arouca, da Fundação Oswaldo Cruz (Ensp/Fiocruz), e aprovado com o parecer n. $32.012 \mathrm{em}$ 06/06/2012. 
A matriz proposta para análise da capacidade de 'coordenação do cuidado', de acordo com as respostas das EqABs e usuários, (re)categorizou indicadores presentes nos instrumentos de coleta do PMAQ-AB, em consonância com revisão de literatura e evidências empíricas. Considerou-se como macroestratégias para consolidação desse atributo 'o fortalecimento da APS e a integração da Rede de Atenção à Saúde'. A primeira macroestratégia foi composta por quatro dimensões e a segunda por três, as quais abrangiam um conjunto de variáveis que caracterizavam a coordenação do cuidado (Quadro 1).

Quadro 1

Dimensões e variáveis para análise da coordenação do cuidado, segundo os respondentes do PMAQ-AB, primeiro ciclo, município do Rio de Janeiro, 2012

\begin{tabular}{|c|c|c|c|c|}
\hline Estratégias & Dimensões & Variáveis & $\begin{array}{l}\text { Profissional } \\
\text { (Módulo II) }\end{array}$ & $\begin{array}{l}\text { Profissional } \\
\text { (Módulo II) }\end{array}$ \\
\hline \multirow[t]{8}{*}{ Fortalecimento da APS } & Organização da & Acolhimento & $\mathrm{x}$ & $\mathrm{x}$ \\
\hline & porta de entrada & Atendimento à demanda espontânea & $x$ & $x$ \\
\hline & pela APS & Atendimento de urgência/emergência & $x$ & $x$ \\
\hline & Resolutividade & Oferta de ações pela EqAB & $\mathrm{x}$ & $\mathrm{x}$ \\
\hline & da APS & Apoio de outros profissionais & $x$ & - \\
\hline & Continuidade do & Atendimento pelo mesmo profissional & - & $x$ \\
\hline & cuidado na APS & Registro das informações & $x$ & $x$ \\
\hline & $\begin{array}{l}\text { Integração } \\
\text { horizontal }\end{array}$ & Reunião de equipe & $x$ & - \\
\hline Integração da Rede de & Organização dos & Agendamento para a rede de referência & $x$ & $x$ \\
\hline \multirow[t]{5}{*}{ Atenção à Saúde } & $\begin{array}{l}\text { fluxos e acesso à } \\
\text { rede de referência }\end{array}$ & $\begin{array}{l}\text { Monitoramento das filas/tempos de espera para } \\
\text { atenção especializada }\end{array}$ & $x$ & - \\
\hline & Continuidade & Prontuário compartilhado & $\mathrm{x}$ & - \\
\hline & informacional & Protocolos clínicos & $\mathrm{x}$ & - \\
\hline & Comunicação entre & Frequência do contato interprofissional & $x$ & - \\
\hline & profissionais & Instrumentos para comunicação interprofissional & $\mathrm{x}$ & - \\
\hline
\end{tabular}

Fonte: Os autores. 


\section{O contexto}

O Rio de Janeiro é a segunda capital mais populosa do Brasil. Dada a sua posição histórica no desenvolvimento do país, reuniu uma importante rede de serviços de saúde, particularmente de unidades hospitalares, com a coordenação das diferentes esferas de governo. Na década de 1990, a rede municipal de AB estava organizada de acordo com modelo tradicional de atenção, e por duas décadas as sucessivas gestões setoriais resistiram em promover a reforma e a substituição desse modelo pela da ESF (Lima, 2014).

Baseada na Estratégia Saúde da Família, a estruturação da rede de atenção básica só foi impulsionada em 2009, o que se constituiu num caso de expansão tardia e com reduzido tempo de implantação. Enquanto Belo Horizonte (MG) e Florianópolis (SC), em 2008, alcançavam uma cobertura pela ESF em torno de 70\% da população, o Rio de Janeiro não chegava a 4\% (Soranz, 2012).

O processo de reforma da atenção primária, iniciado em 2009, incluiu a definição e a articulação da rede segundo a lógica dos territórios integrados de saúde e promoveu uma importante expansão do número de UBSs, com a implantação de mais de setecentas novas equipes de saúde da família até 2012, consolidando assim o modelo da ESF na cidade (Soranz, 2012). Segundo dados de agosto de 2015, o município possuía cobertura de $45,5 \%$ da população e 843 equipes de saúde da família (Brasil, 2015a).

Harzheim (2013, p. xii), em publicação referente à 'avaliação da implantação das clínicas da família no Rio de Janeiro', destaca que, além do aumento de cobertura no período, houve uma aposta na qualidade das unidades de saúde, que concentraram mais equipes com estrutura física diferenciada e incorporação de tecnologia de apoio diagnóstico apropriada à prática da $\mathrm{AB}$.

Ao se considerarem, portanto, as particularidades da implantação da ESF na cidade, pretendeu-se cotejar os resultados obtidos pelo PMAQ-AB no âmbito municipal com a experiência nacional e outros estudos de igual natureza, visando contribuir para a implantação de estratégias e discussão de uma matriz de análise para a coordenação do cuidado, sensível às especificidades do Sistema Único de Saúde (SUS) e que reflita avanços e lacunas na oferta de cuidado integral em saúde.

\section{Estratégias para fortalecimento da atenção primária à saúde}

Para a apresentação dos resultados referentes ao fortalecimento da APS, foram utilizados os dados da Tabela 1 (EqAB) e parte da Tabela 3 (usuários), composta pelas dimensões: organização da porta de entrada pela APS, resolutividade da APS, continuidade do cuidado na APS e integração horizontal. 


\section{Organização da porta de entrada pela APS}

A organização da porta de entrada é um dos elementos essenciais para potencializar a APS como coordenadora do cuidado e ordenadora das redes de atenção à saúde. De acordo com o Quadro 1, está composta pelo seguinte conjunto de indicadores: acolhimento, atendimento à demanda espontânea e atendimento de urgência e emergência.

No município, 99\% das EqABs relataram ter acolhimento implantado, com frequência de cinco dias por semana para a grande maioria $(89,4 \%)$, informação ratificada pelos usuários, os quais informaram ser escutados na UBSs sem hora marcada $(88,1 \%)$. O enfermeiro $(98,4 \%)$ e o agente comunitário de saúde (ACS) (95,0\%) foram citados como os profissionais que mais participaram do acolhimento, segundo as EqABs, embora a participação do profissional médico também tenha sido expressiva (Tabela 1). A avaliação dos usuários apontou que o ACS é o profissional mais envolvido no acolhimento, seguido pelo enfermeiro e pelo médico (Tabela 3).

Das 324 equipes que compuseram o universo da pesquisa, $83 \%$ informaram possuir protocolos com definição de diretrizes terapêuticas para acolhimento à demanda espontânea ou urgência, sendo a principal ação desencadeada a marcação na agenda do médico $(97,5 \%)$ e do dentista $(74,1 \%)$ (Tabela 1). Na avaliação de profissionais e usuários, o dentista foi apontado como o profissional que menos frequentemente se envolvia nas ações de acolhimento implementadas pela EqAB.

A quase totalidade das EqABs relatou reservar vagas para atendimento no mesmo dia. Apesar de os profissionais afirmarem a existência de acolhimento e vagas reservadas para atendimento à demanda espontânea diariamente, essa informação não foi confirmada pelos usuários entrevistados. O percentual de usuários $(18,1 \%)$ que afirmou ser possível marcar consulta para o mesmo dia foi baixo (Tabela 3), indicando divergências em relação à percepção dos profissionais, que apresentaram avaliações mais positivas (Tabela 1).

A marcação de consultas por meio do ACS foi reportada por $48 \%$ de usuários, enquanto $45,5 \%$ procuraram diretamente a UBS, sendo praticamente inexistente a marcação por outras vias. O tempo de espera por uma consulta, para os casos não urgentes, mostrou-se superior a 21 dias para $33,8 \%$ dos profissionais entrevistados e de até dez dias para $39,2 \%$.

Foi pouco frequente o monitoramento do tempo de espera por uma consulta na unidade, segundo profissionais da EqAB. Embora percentual considerável das equipes tenha mencionado a realização de estudos da demanda espontânea nos últimos 12 meses, a comprovação por meio de documentos revelou-se baixa (Tabela 1).

A procura pela UBS para o atendimento de urgência ou emergência foi relatada por $73 \%$ dos usuários que necessitaram desse tipo de atendimento, 
constituindo-se no serviço mais procurado, em detrimento de hospitais públicos e serviços de pronto atendimento. Destes, $84,2 \%$ foram atendidos na mesma hora, sem consulta marcada, tendo recebido atendimento médico em $63,8 \%$ dos casos e em 27,7\% de enfermagem (Tabela 3). Entre as EqABs, 96,6\% dos respondentes relataram absorver a demanda de urgência/emergência. A maioria dos profissionais da equipe afirmou ter recebido capacitação para avaliação e classificação de risco e vulnerabilidade e utilizar essa estratégia no acolhimento, embora nem sempre seu uso fosse capaz de definir a resposta ao usuário (Tabela 1).

\section{Tabela 1}

Coordenação do cuidado: indicadores referentes às variáveis da estratégia para fortalecimento da atenção primária à saúde, segundo as EqABs participantes do PMAQ-AB, primeiro ciclo, município do Rio de Janeiro, 2012

\begin{tabular}{|c|c|c|}
\hline Indicadores & $\begin{array}{c}\mathrm{N} \\
(324)\end{array}$ & $\begin{array}{c}\% \\
(100)\end{array}$ \\
\hline \multicolumn{3}{|l|}{ Acolhimento } \\
\hline EqAB com acolhimento implantado & 322 & 99,4 \\
\hline Frequência do acolhimento: cinco dias por semana & $288(322)$ & 89,4 \\
\hline Profissionais que participam do acolhimento: & 322 & 100 \\
\hline Enfermeiro & 317 & 98,4 \\
\hline ACS & 306 & 95,0 \\
\hline Médico & 247 & 76,7 \\
\hline Técnico de enfermagem & 243 & 75,5 \\
\hline Odontólogo & 155 & 48,1 \\
\hline $\begin{array}{l}\text { EqAB que possui protocolos com definição de diretrizes terapêuticas para acolhimento à } \\
\text { demanda espontânea/urgência }\end{array}$ & $269(324)$ & 83,0 \\
\hline Ações desencadeadas a partir do acolhimento: & 324 & 100 \\
\hline Marcação de usuários na agenda do médico & 316 & 97,5 \\
\hline Marcação de usuários na agenda do dentista & 240 & 74,1 \\
\hline Marcação de usuários na agenda do enfermeiro & 3 & 0,9 \\
\hline \multicolumn{3}{|l|}{ Atendimento à demanda espontânea } \\
\hline UBS com agendamento de consultas em qualquer dia da semana, em qualquer horário & $266(324)$ & 82,1 \\
\hline EqABs que reservam vagas para atendimento no mesmo dia & $316(324)$ & 97,5 \\
\hline Existência de documento que comprove & $280(316)$ & 88,6 \\
\hline EqABs que controlam o tempo de espera para atendimento dos usuários & $29(316)$ & 9,1 \\
\hline EqABs que agendam consultas para não integrantes de grupos prioritários em qualquer dia & $293(324)$ & 90,4 \\
\hline Tempo de espera para usuários não atendidos no mesmo dia: & 293 & 100 \\
\hline Até 5 dias & 27 & 9,2 \\
\hline De 6 a 10 dias & 88 & 30,0 \\
\hline De 11 a 20 dias & 79 & 27,0 \\
\hline Mais de 21 dias & 99 & 33,8 \\
\hline EqABs que realizaram estudo da demanda espontânea nos últimos 12 meses & 209 & 64,5 \\
\hline Existência de documento que comprove & $152(209)$ & 72,7 \\
\hline
\end{tabular}




\section{Continuação - Tabela 1}

Coordenação do cuidado: indicadores referentes às variáveis da estratégia para fortalecimento da atenção primária à saúde, segundo as EqABs participantes do PMAQ-AB, primeiro ciclo, município do Rio de Janeiro, 2012

\begin{tabular}{|c|c|c|}
\hline Indicadores & $\underset{(324)}{N}$ & $\begin{array}{c}\% \\
(100)\end{array}$ \\
\hline \multicolumn{3}{|l|}{ Atendimento de urgência/emergência } \\
\hline EqABs que realizam atendimento de urgência/emergência & $313(324)$ & 96,6 \\
\hline $\begin{array}{l}\text { Profissionais da EqAB capacitados para avaliação e classificação de risco e vulnerabilidade } \\
\text { dos usuários }\end{array}$ & $250(324)$ & 77,2 \\
\hline EqABs que realizam avaliação de risco e vulnerabilidade no acolhimento dos usuários & $308(324)$ & 95,1 \\
\hline $\begin{array}{l}\text { EqABs que têm condições de definir a resposta com base na avaliação de risco e } \\
\text { vulnerabilidade no acolhimento dos usuários }\end{array}$ & $245(308)$ & 79,5 \\
\hline \multicolumn{3}{|l|}{ Oferta de ações pela EqAB } \\
\hline EqABs que realizam procedimentos/pequenas cirurgias & $297(324)$ & 91,7 \\
\hline Procedimentos realizados: & 297 & 100 \\
\hline Curativos & 294 & 99,0 \\
\hline Retirada de pontos & 291 & 98,0 \\
\hline Nebulização/inalação & 290 & 97,6 \\
\hline Drenagem de abscesso & 254 & 85,5 \\
\hline Sutura de ferimentos & 231 & 77,8 \\
\hline Lavagem de ouvido & 183 & 61,6 \\
\hline Extração de unha & 169 & 56,9 \\
\hline EqABs que realizam coleta de material para exames laboratoriais & $322(324)$ & 99,4 \\
\hline EqABs que recebem medicamentos suficientes da farmácia básica & $262(324)$ & 80,9 \\
\hline \multicolumn{3}{|l|}{ Apoio de outros profissionais } \\
\hline EqABs que participaram do Telessaúde no último ano & $59(324)$ & 18,2 \\
\hline Utilização do Telessaúde: & 59 & 100 \\
\hline Segunda opinião formativa & 26 & 44,1 \\
\hline Teleconsultoria & 21 & 35,6 \\
\hline Telediagnóstico & 12 & 20,3 \\
\hline $\begin{array}{l}\text { EqABs que recebem apoio de outros profissionais para auxiliar ou apoiar na resolução de } \\
\text { casos complexos }\end{array}$ & $314(324)$ & 96,3 \\
\hline Frequência do apoio matricial: & 314 & 100 \\
\hline Semanal & 180 & 57,3 \\
\hline Sem periodicidade definida & 66 & 21,0 \\
\hline Profissionais que participam do apoio matricial mais frequentemente: & 314 & 100 \\
\hline Psicólogo & 234 & 74,5 \\
\hline Fisioterapeuta & 162 & 51,6 \\
\hline Nutricionista & 153 & 48,7 \\
\hline Psiquiatra & 118 & 37,6 \\
\hline Assistente social & 114 & 36,3 \\
\hline Educador físico & 105 & 33,4 \\
\hline Pediatra & 100 & 31,8 \\
\hline Ginecologista/obstetra & 93 & 29,6 \\
\hline
\end{tabular}


Continuação - Tabela 1

Coordenação do cuidado: indicadores referentes às variáveis da estratégia para fortalecimento da atenção primária à saúde, segundo as EqABs participantes do PMAQ-AB, primeiro ciclo, município do Rio de Janeiro, 2012

\begin{tabular}{|c|c|c|}
\hline Indicadores & $\begin{array}{c}\mathrm{N} \\
(324)\end{array}$ & $\begin{array}{c}\% \\
(100)\end{array}$ \\
\hline Fonoaudiólogo & 75 & 23,9 \\
\hline Farmacêutico & 40 & 12,7 \\
\hline Ações de apoio matricial: & 314 & 100 \\
\hline Discussão de casos clínicos, eventos sentinelas, casos difíceis & 297 & 94,6 \\
\hline Consultas clínicas de pacientes & 287 & 91,4 \\
\hline Ações clínicas compartilhadas com os profissionais da EqAB & 281 & 89,5 \\
\hline Construção conjunta de projetos terapêuticos com os profissionais da EqAB & 261 & 83,1 \\
\hline Visitas com os profissionais da EqAB & 256 & 81,5 \\
\hline Atividades de educação permanente conjuntamente com os profissionais da EqAB & 220 & 70,1 \\
\hline Discussões sobre o processo de trabalho da EqAB e do apoio matricial & 33 & 10,5 \\
\hline \multicolumn{3}{|l|}{ Registro das informações } \\
\hline EqAB com prontuário eletrônico & $310(324)$ & 95,7 \\
\hline Informações contidas nos prontuários: & 324 & 100 \\
\hline Hipótese diagnóstica/problema ou condição & 292 & 90,1 \\
\hline Exames solicitados & 279 & 86,1 \\
\hline \multicolumn{3}{|l|}{ Reunião de equipe } \\
\hline EqABs que realizam reunião de equipe semanalmente & $307(322)$ & 95,3 \\
\hline Temas da reunião de equipe: & 322 & 100 \\
\hline Discussão de casos pela equipe, eventos sentinelas, casos difíceis & 319 & 99,1 \\
\hline Construção/discussão de projeto terapêutico singular & 266 & 82,6 \\
\hline Qualificação clínica com participação de equipes de apoio matricial (NASF,CAPS, especialidades) & 246 & 76,4 \\
\hline
\end{tabular}

Fonte: PMAQ-AB (Brasil, 2015b).

Nota: EqAB - Equipe de atenção básica; UBS - Unidade básica de saúde; NASF - Núcleo de Apoio à Saúde da Família; CAPS - Centro de Atenção Psicossocial.

\section{Resolutividade da APS}

Outra dimensão diretamente relacionada ao fortalecimento da APS foi a capacidade de as equipes produzirem respostas oportunas às necessidades dos usuários, por meio da ampliação e qualificação das ações ofertadas.

Além dos atendimentos esperados para um serviço de APS, a quase totalidade das UBS coletava material para exames laboratoriais e realizava procedimentos e pequenas cirurgias $(91,7 \%)$. Os mais frequente eram: curativos, retirada de pontos, nebulização/inalação e drenagem de abscesso; e os menos frequentes, a sutura de ferimentos, lavagem de ouvido e extração de unhas (Tabela 1). Na avaliação dos usuários, 76,2\% relataram que a EqAB sempre buscou resolver os problemas sem que eles precisassem se deslocar para outro ponto de atenção. 
Quanto à disponibilidade de medicamentos, 80,9\% das equipes relataram recebê-los em quantidade suficiente. Entretanto, somente 47,3\% dos usuários registraram que os fármacos prescritos pela EqAB estavam sempre disponíveis. Os usuários também enfrentaram barreiras para saber os resultados dos exames na UBS (Tabela 3).

A existência de apoio matricial, como estratégia para qualificação das equipes por meio de trocas sistemáticas com profissionais de outras especialidades, sobretudo para a resolução de casos complexos, foi referida pela quase totalidade dos entrevistados. A periodicidade mais frequente era a semanal. O psicólogo foi o profissional mais envolvido, seguido pelo fisioterapeuta e o nutricionista. As atividades desenvolvidas no âmbito do apoio matricial incluíram discussão de casos clínicos, eventos sentinelas, casos difíceis; consultas clínicas de pacientes; e ações clínicas compartilhadas. Discussões sobre o processo de trabalho da EqAB e do próprio apoio matricial foram pouco frequentes (Tabela 1).

Outras tecnologias com potencialidade para aumentar a resolutividade e qualificar as ações da APS por meio do uso de tecnologias de informação e comunicação (TICs), como o Telessaúde, por exemplo, foram subutilizadas pelas EqABs $(18,2 \%)$ (Tabela 1$)$.

\section{Organização da porta de entrada pela APS}

A continuidade do cuidado na APS é uma dimensão primordial para constituição do vínculo e resolutividade e foi aqui analisada por meio da frequência de atendimento pelo mesmo profissional da EqAB e registro das informações. Dos usuários entrevistados, $81,3 \%$ afirmaram que eram atendidos pelo mesmo médico sempre ou na maioria das vezes, reduzindo o percentual quando se tratava do enfermeiro $(68,5 \%)$ (Tabela 3$)$.

Sobre os registros clínicos na APS, a grande maioria das EqABs no município trabalhava com o prontuário eletrônico, no qual os registros mais frequentes foram a hipótese diagnóstica/problema ou condição e os exames solicitados (Tabela 1). De acordo com os usuários, a maior parte dos profissionais das EqABs $(88 \%)$ realizava anotações no prontuário após a consulta, embora aproximadamente $1 / 3$ tenha relatado que o profissional nunca se lembrava do que acontecera nas últimas consultas (Tabela 3).

\section{Integração horizontal}

Uma das dimensões da coordenação referiu-se a estratégias que possibilitassem a integração entre serviços no âmbito da própria APS e no nível local, 
em um processo de coordenação horizontal. No município, 95,3\% das equipes afirmaram promover reunião semanal, sobretudo para discussão de casos complexos, construção de projetos terapêuticos singulares e realização de qualificações promovidas pelas equipes de apoio matricial (Tabela 1).

\section{Estratégias para integração da Rede de Atenção à Saúde (RAS)}

Os resultados que seguem referem-se à integração da RAS, e os dados utilizados pertencem à Tabela 2 (EqAB) e parte da Tabela 3 (usuários), incluindo as seguintes dimensões: organização dos fluxos e acesso à rede de referência, continuidade informacional e comunicação entre profissionais.

\section{Organização dos fluxos e acesso à rede de referência}

Ao se tomar como foco essa dimensão, entende-se que uma APS coordenadora do cuidado deve ser capaz de realizar o agendamento para sua rede de referência e de monitorar as filas e os tempos de espera para atenção especializada, desde que tenha à disposição, por exemplo, instrumentos que permitam a integração assistencial, como centrais de regulação/marcação e prontuários eletrônicos compartilhados.

A quase totalidade dos respondentes indicou a existência de central de marcação para outros pontos de atenção, sobretudo para consultas $(99,7 \%) \mathrm{e}$ exames especializados $(97,8 \%)$. O percentual para a marcação de leitos é comparativamente mais baixo $(40,7 \%)$ (Tabela 2$)$. A maioria das equipes dispunha de documentos contendo as indicações das referências e dos fluxos pactuados pela gestão municipal por área de abrangência.

De acordo com os profissionais, a forma mais frequente de agendamento para consultas especializadas foi a marcação realizada pela UBS, com data posteriormente comunicada ao usuário $(95,7 \%)$, percentual que diferiu do relatado pelos usuários, os quais identificaram essa forma de agendamento em 57, $1 \%$ dos casos. Cerca de $1 / 5$ dos profissionais entrevistados mencionaram que o usuário pode receber uma ficha de referência e ser orientado para buscar o agendamento, em serviço ou profissional determinado, de forma individualizada (Tabela 2). Essa forma de agendamento foi confirmada por 13\% dos usuários (Tabela 3). A definição prévia de maternidade para as gestantes acompanhadas foi relatada por 99,7\% dos respondentes (Tabela 2). 
Tabela 2

Coordenação do cuidado: indicadores referentes às variáveis da estratégia de integração da Rede de Atenção à Saúde, segundo as EqABs do PMAQ-AB, primeiro ciclo, município do Rio de Janeiro, 2012

\begin{tabular}{|c|c|c|}
\hline Indicadores & $\underset{(324)}{N}$ & $\begin{array}{c}\% \\
(100)\end{array}$ \\
\hline \multicolumn{3}{|l|}{ Agendamento para a rede de referência } \\
\hline Centrais de marcação disponíveis para as EqABs: & 324 & 100 \\
\hline Consultas especializadas & 323 & 99,7 \\
\hline Exames especializados & 317 & 97,8 \\
\hline Leitos & 192 & 59,3 \\
\hline EqABs que possuem documentos com referências e fluxos do território & $267(324)$ & 82,4 \\
\hline Formas de agendamento para consultas especializadas: & 324 & 100 \\
\hline Consulta agendada na UBS e data informada na mesma hora & 58 & 17,9 \\
\hline Consulta agendada na UBS e data posteriormente informada & 310 & 95,7 \\
\hline Recebe uma ficha de referência para serviço/profissional especializado determinado & 65 & 20,1 \\
\hline Recebe uma ficha de referência para algum serviço/profissional especializado & 33 & 10,2 \\
\hline EqABs que possuem maternidade definida para as gestantes acompanhadas & $323(324)$ & 99,7 \\
\hline \multicolumn{3}{|l|}{ Monitoramento das filas/tempos de espera para atenção especializada } \\
\hline EqABs que mantêm registro dos usuários de maior risco referenciados & $223(324)$ & 68,8 \\
\hline Existência de documento que comprove & $183(223)$ & 82,1 \\
\hline \multicolumn{3}{|l|}{ Tempo de espera estimado para consultas especializadas: } \\
\hline Cardiologia: & 155 & 100 \\
\hline Até 30 dias & 29 & 19 \\
\hline De 31 a 60 dias & 26 & 17 \\
\hline De 61 a 90 dias & 40 & 26 \\
\hline Mais de 91 dias & 60 & 39 \\
\hline Ortopedia: & 196 & 100 \\
\hline Até 30 dias & 36 & 18 \\
\hline De 31 a 60 dias & 15 & 8 \\
\hline De 61 a 90 dias & 49 & 25 \\
\hline Mais de 91 dias & 96 & 49 \\
\hline Oftalmologia: & 215 & 100 \\
\hline Até 30 dias & 28 & 13 \\
\hline De 31 a 60 dias & 13 & 6 \\
\hline De 61 a 90 dias & 43 & 20 \\
\hline Mais de 91 dias & 131 & 61 \\
\hline Mamografia para diagnóstico precoce do câncer da mama: & 156 & 100 \\
\hline Até 30 dias & 85 & 55 \\
\hline De 31 a 60 dias & 28 & 18 \\
\hline De 61 a 90 dias & 22 & 15 \\
\hline Mais de 91 dias & 21 & 13 \\
\hline Monitoramento das listas de espera para: & 324 & 100 \\
\hline Hipertensão arterial sistêmica & 201 & 62,0 \\
\hline Existência de documento que comprove & $149(201)$ & 74 \\
\hline Diabetes mellitus & 205 & 63,3 \\
\hline
\end{tabular}


Cotinuação - Tabela 2

Coordenação do cuidado: indicadores referentes às variáveis da estratégia de integração da Rede de Atenção à Saúde, segundo as EqABs do PMAQ-AB, primeiro ciclo, município do Rio de Janeiro, 2012

\begin{tabular}{|c|c|c|}
\hline Indicadores & $\begin{array}{c}\mathrm{N} \\
(324)\end{array}$ & $\begin{array}{c}\% \\
(100)\end{array}$ \\
\hline \multicolumn{3}{|l|}{ Prontuário compartilhado } \\
\hline Prontuário eletrônico compartilhado com outros pontos de atenção & $87(310)$ & $87(310)$ \\
\hline \multicolumn{3}{|l|}{ Protocolos clínicos } \\
\hline \multicolumn{3}{|l|}{ EqABs que possuem protocolos para: } \\
\hline Câncer do colo de útero & 305 & 94,1 \\
\hline Hipertensão arterial & 304 & 93,8 \\
\hline Diabetes mellitus & 303 & 93,5 \\
\hline Saúde mental & 241 & 74,4 \\
\hline Álcool e drogas & 213 & 65,7 \\
\hline EqABs que possuem protocolos com definição de prioridades para referência & $260(324)$ & 80,2 \\
\hline \multicolumn{3}{|l|}{ Frequência do contato interprofissional } \\
\hline \multicolumn{3}{|l|}{ Frequência do contato de profissionais da EqAB com especialistas para troca de } \\
\hline informações sobre usuários referenciados: & 324 & 100 \\
\hline Sempre & 35 & 10,8 \\
\hline Algumas vezes & 176 & 54,3 \\
\hline Nunca & 113 & 34,9 \\
\hline \multicolumn{3}{|l|}{ Frequência de contato dos especialistas com profissionais da EqAB para troca de } \\
\hline informações sobre usuários referenciados: & 324 & 100 \\
\hline Sempre & 14 & 4,3 \\
\hline Algumas vezes & 139 & 42,9 \\
\hline Nunca & 171 & 52,8 \\
\hline \multicolumn{3}{|l|}{ Instrumentos para comunicação interprofissional } \\
\hline Existência de fluxo de comunicação institucionalizado & $176(324)$ & 54,3 \\
\hline Tipo de fluxo institucional: & 176 & 100 \\
\hline Discussão de casos & 40 & 22,7 \\
\hline Reuniões técnicas com os especialistas da rede & 26 & 14,8 \\
\hline Teleconferência & 6 & 3,4 \\
\hline Telessaúde & 10 & 5,7 \\
\hline Prontuário eletrônico & 21 & 11,9 \\
\hline Comunicação eletrônica & 44 & 25,0 \\
\hline Ficha de referência/contrarreferência com história clínica e sugestões de conduta & 149 & 84,7 \\
\hline Canal de comunicação disponível na UBS: & 176 & 100 \\
\hline Telefone & 153 & 86,9 \\
\hline Internet & 114 & 64,8 \\
\hline UBSs com lista de contato com especialistas da rede SUS & $213(324)$ & 65,7 \\
\hline Existência de documento que comprove & $181(213)$ & 85,0 \\
\hline
\end{tabular}

Fonte: PMAQ-AB (Brasil, 2015b).

Nota: EqABs - Equipes de atenção básica; UBS - Unidade básica de saúde. 
Cerca de $60 \%$ dos usuários afirmaram que as equipes sempre conseguiam marcar consulta com especialista ou outro profissional, embora 17,6\% tivessem afirmado que nunca era possível (Tabela 3). Este último percentual esteve próximo da forma de marcação reportada pelos profissionais, na qual o usuário recebia a guia de referência e buscava individualmente pelo serviço/profissional especializado.

Ao se considerar que a referência é um procedimento meio para o acesso do usuário aos serviços ou especialistas, o monitoramento do seu percurso constitui parte fundamental do cuidado. Aproximadamente $70 \%$ dos profissionais informaram que as equipes mantinham registro dos usuários de maior risco encaminhados; destes, 82,1\% comprovaram a prática (Tabela 2). Entretanto, quando os usuários foram perguntados se a equipe conversava sobre o atendimento realizado fora da UBS, 55,1\% dos entrevistados afirmaram que nunca, e somente $33 \%$ responderam que sempre (Tabela 3).

Quando analisadas as quatro especialidades/serviços com maior número de encaminhamentos (cardiologia, ortopedia, oftalmologia e mamografia), observou-se que os tempos de espera para marcação eram invariavelmente longos, exceto para pedido de mamografia. Um grande percentual de usuários aguardava mais de noventa dias para uma consulta com médico de qualquer uma das três especialidades listadas. Para o exame de mamografia, 55\% das mulheres conseguiam em até 15 dias, e somente em torno de $10 \%$ aguardavam mais de noventa dias, segundo percepção dos profissionais (Tabela 2).

Tabela 3

Coordenação do cuidado: indicadores referentes às variáveis das estratégias de fortalecimento da atenção primária à saúde e integração da Rede de Atenção à Saúde, segundo usuários participantes do PMAQ-AB, primeiro ciclo, município do Rio de Janeiro, 2012

\begin{tabular}{lcc}
\hline \multicolumn{1}{c}{ Indicadores } & $\mathbf{N}$ & $\%$ \\
& $(1.313)$ & $(100)$ \\
\hline Acolhimento & & \\
Usuários que conseguem ser escutados na UBS sem hora marcada, na maioria das vezes & $991\left(1.125^{*}\right)$ & 88,1 \\
Profissionais que realizam a escuta: & 991 & 100 \\
Médico & 426 & 43,0 \\
Enfermeiro & 469 & 47,3 \\
Cirurgião-dentista & 34 & 3,4 \\
ACS & 649 & 65,5 \\
Atendimento à demanda espontânea & & 100 \\
Principais formas para marcação de consulta na UBS:** & 1.313 & 13 \\
O usuário vai à UBS e marca o atendimento a qualquer hora & 597 & 45,5 \\
O ACS marca a consulta & 630 & 48,0 \\
\end{tabular}


Cotinuação - Tabela 3

Coordenação do cuidado: indicadores referentes às variáveis das estratégias de fortalecimento da atenção primária à saúde e integração da Rede de Atenção à Saúde, segundo usuários participantes do PMAQ-AB, primeiro ciclo, município do Rio de Janeiro, 2012

\begin{tabular}{|c|c|c|}
\hline Indicadores & $\begin{array}{c}\mathrm{N} \\
(1.313)\end{array}$ & $\begin{array}{c}\% \\
(100)\end{array}$ \\
\hline Dias e horários para marcação de consulta na UBS: & 1.313 & 100 \\
\hline Todos os dias da semana & 958 & 73,0 \\
\hline Não sabe/não informou & 141 & 10,7 \\
\hline A qualquer hora do dia & 404 & 30,8 \\
\hline Somente em alguns horários & 107 & 8,1 \\
\hline Usuários que conseguem marcar consulta para o mesmo dia & $237(1.313)$ & 18,1 \\
\hline $\begin{array}{l}\text { Presença do médico na UBS/atividades no bairro durante todos os horários de } \\
\text { funcionamento da unidade }\end{array}$ & $881(1.313)$ & 67,1 \\
\hline \multicolumn{3}{|l|}{ Atendimento de urgência/emergência } \\
\hline $\begin{array}{l}\text { Usuários que procuraram atendimento na última vez que tiveram um problema de saúde } \\
\text { de urgência }\end{array}$ & $857(933)^{* * *}$ & 91,2 \\
\hline Principais serviços de saúde procurados: & 857 & 100 \\
\hline UBS & 626 & 73,0 \\
\hline Hospital público & 61 & 7,1 \\
\hline Pronto atendimento 24 horas & 100 & 11,7 \\
\hline Usuários que conseguiram ser atendidos na UBS na mesma hora, sem consulta marcada & $527(626)$ & 84,2 \\
\hline Profissional responsável pelo atendimento: & 527 & 100 \\
\hline Médico & 336 & 63,8 \\
\hline Enfermeiro & 146 & 27,7 \\
\hline Auxiliar/técnico de enfermagem & 10 & 1,9 \\
\hline Outro & 26 & 4,9 \\
\hline Não sabe/não respondeu & 9 & 1,7 \\
\hline \multicolumn{3}{|l|}{ Oferta de ações pela EqAB } \\
\hline EqAB sempre busca resolver as necessidades/problemas na própria UBS & $1.001(1.313)$ & 76,2 \\
\hline Usuários relataram facilidade para saber os resultados dos exames na UBS: & 1.313 & 100 \\
\hline Sempre & 629 & 47,9 \\
\hline Algumas vezes & 237 & 18,1 \\
\hline Nunca & 444 & 33,8 \\
\hline Não sabe/não respondeu & 3 & 0,2 \\
\hline Disponibilidade de medicamentos prescritos pela EqAB: & 1.313 & 100 \\
\hline Sempre & 621 & 47,3 \\
\hline Algumas vezes & 616 & 46,9 \\
\hline Nunca & 32 & 2,4 \\
\hline Não sabe/não respondeu & 44 & 3,4 \\
\hline \multicolumn{3}{|l|}{ Atendimento pelo mesmo profissional } \\
\hline Usuários atendidos pelo mesmo médico sempre/na maioria das vezes & 1.068 & 81,3 \\
\hline Usuários atendidos pelo mesmo enfermeiro sempre/na maioria das vezes & 900 & 68,5 \\
\hline
\end{tabular}


Coordenação do cuidado: indicadores referentes às variáveis das estratégias de fortalecimento da atenção primária à saúde e integração da Rede de Atenção à Saúde, segundo usuários participantes do PMAQ-AB, primeiro ciclo, município do Rio de Janeiro, 2012

\begin{tabular}{|c|c|c|}
\hline Indicadores & $\underset{(1.313)}{\mathrm{N}}$ & $\begin{array}{c}\% \\
(100)\end{array}$ \\
\hline \multicolumn{3}{|l|}{ Registro das informações } \\
\hline Profissionais da EqAB fazem anotações no prontuário após consulta & $1.156(1.313)$ & 88,0 \\
\hline Profissionais da EqAB se lembram do que aconteceu nas últimas consultas: & 1.313 & 100 \\
\hline Sempre & 561 & 42,7 \\
\hline Algumas vezes & 235 & 17,9 \\
\hline Nunca & 410 & 31,2 \\
\hline Não sabe/não respondeu & 107 & 8,1 \\
\hline \multicolumn{3}{|l|}{ Agendamento para a rede de referência } \\
\hline EqAB consegue marcar consulta com outros profissionais ou especialistas: & $601^{* * * *}$ & 100 \\
\hline Sempre & 363 & 60,4 \\
\hline Algumas vezes & 118 & 19,6 \\
\hline Nunca & 106 & 17,6 \\
\hline Não sabe/não respondeu & 14 & 2,4 \\
\hline Principais formas de agendamento para consulta especializada** & $601^{* * * *}$ & 100 \\
\hline Sai da UBS com a consulta agendada & 92 & 15,3 \\
\hline Marcada pela UBS e a data posteriormente informada & 343 & 57,1 \\
\hline Recebe uma ficha de referência e procura o serviço indicado pelo profissional & 78 & 13,0 \\
\hline EqAB conversa com o usuário após atendimento realizado fora da UBS & $601^{* * * *}$ & 100 \\
\hline Sempre & 200 & 33,3 \\
\hline Algumas vezes & 67 & 11,1 \\
\hline Nunca & 331 & 55,1 \\
\hline Não sabe/não respondeu & 3 & 0,5 \\
\hline
\end{tabular}

Fonte: PMAQ-AB (Brasil, 2015b).

Nota: EqABs - Equipes de atenção básica; ACS - Agente comunitário de saúde; UBS - Unidade básica de saúde.

* Número de usuários que precisou ir à UBS sem hora marcada $=1.125$.

* Registraram-se somente as formas mais frequentes.

*** Número de usuários que necessitaram de atendimento de urgência $=933$.

**** Número de usuários que necessitaram de consulta com especialista $=601$.

\section{Continuidade informacional}

Outro elemento facilitador da coordenação do cuidado foi a existência de prontuário informatizado no âmbito da APS (Tabela 1). Contudo, o fato de não estar integrado às demais unidades da rede limitava as possibilidades de coordenação do cuidado. Somente $28,1 \%$ das EqABs registraram que havia compartilhamento com outros pontos de atenção (Tabela 2).

A existência de protocolos clínicos, disponíveis para que as equipes tomassem decisão sobre a priorização de casos para a referência, foi relatada 
por $80,2 \%$ dos profissionais. Protocolos clínicos para câncer do colo do útero, hipertensão arterial e diabetes foram bastante frequentes, mas os menos encontrados para saúde mental e álcool e drogas (Tabela 2).

\section{Comunicação entre profissionais da rede}

O diálogo entre os profissionais da rede por meio da utilização de ferramentas de comunicação favorece uma resposta mais rápida e efetiva no cuidado ao usuário, aumentando a resolutividade e evitando encaminhamentos desnecessários.

Aproximadamente metade dos profissionais entrevistados afirmou a existência de fluxo de comunicação formal com profissionais de outros pontos de atenção. Ainda assim, 34,9\% dos profissionais da EqAB avaliaram que nunca entraram em contato com especialistas para troca de informações sobre usuários referenciados, sendo maior o percentual de especialistas que nunca entraram em contato com as EqABs. O tipo de comunicação formal mais frequente acontecia por meio da guia de referência e contrarreferência com história clínica e sugestões de conduta. A comunicação eletrônica, a teleconferência e o Telessaúde foram pouco utilizados para comunicação interprofissional. O telefone foi um canal disponível para cerca de $87 \%$ dos profissionais, e a internet, para $64,8 \%$, o que pode representar barreira para a utilização de outras estratégias comunicacionais que exigem conectividade (Tabela 2).

A existência na UBS de lista com os contatos de especialistas da rede SUS foi referida por apenas $65,7 \%$ dos profissionais entrevistados; foi ainda menor o percentual das equipes que comprovaram a existência do documento (Tabela 2).

\section{Discussão}

Ao buscar compreender o papel da APS na coordenação do cuidado no município do Rio de Janeiro, importantes avanços e barreiras foram identificados. Ainda que os resultados do PMAQ-AB não possam ser amplamente generalizados para o conjunto das EqABs do município, o diagnóstico oferecido possibilitou o mapeamento de pontos nodais para o enfretamento de elementos da fragmentação do cuidado.

A implantação do acolhimento e atendimento à demanda espontânea mostrou resultado mais positivo em comparação com a média das EqABs de municípios com mais de quinhentos mil habitantes avaliados no PMAQ-AB (Fausto et al., 2014). Entretanto, a porta de entrada pela APS parece ainda 
não atender às necessidades e expectativas dos usuários, permanecendo expressivo o percentual dos que não conseguiram agendar atendimento para o mesmo dia, em contraposição à avaliação dos profissionais. Monitorar os tempos de espera para consultas na UBS, prática pouco frequente, parece ser necessário enquanto se avança para a garantia de atendimento oportuno para todos que buscam a APS com a ampliação da abertura das agendas locais.

A avaliação dos usuários apontou que o ACS era o profissional que mais realizava o acolhimento dentro da UBS e as marcações de consultas. A carteira de serviços, que norteia as ações da APS no município, define que os ACSs devem participar em dois turnos de uma "escala de atendimento na recepção, com guichês de atendimento exclusivos por equipe" (Rio de Janeiro, 2011, p. 10), o que não é contrário ao contido na PNAB (Brasil, 2012b). Entretanto, vários autores têm problematizado o processo de trabalho dos ACSs em curso na rede municipal de saúde, incluindo o despreparo para a execução do acolhimento à burocratização do trabalho, focado em visitas domiciliares e atividades administrativas como a marcação de consultas, o que poderia comprometer a principal marca e especificidade desse profissional nas EqABs - a inserção territorial e comunitária (Bornstein e David, 2014; Carneiro e Martins, 2015).

Os resultados apontaram baixa participação do dentista na atividade geral de acolhimento, segundo o profissional respondente pela EqAB. Estudo que analisou a seção de saúde bucal do Módulo II, tendo o dentista como respondente, mostrou que $80,6 \%$ realizavam o acolhimento de demanda espontânea (Gabriel e Casotti, 2016), o que sugeriu uma gestão independente da porta de entrada para o atendimento odontológico.

A marcação de consultas médicas foi a ação mais frequente a partir do acolhimento e quase inexistente no caso de agendamento para o profissional de enfermagem, indicando que suas ações estariam concentradas no atendimento à demanda programada e no âmbito da administração/coordenação da UBS, reduzindo a capacidade da equipe na resolução de demandas clínicas individuais. Estudos apontam para um excesso de atividades burocráticas e gerenciais desenvolvidas pelo enfermeiro na $\mathrm{AB}$, que o afasta das ações assistenciais e reduz a capacidade da equipe na resolução das demandas clínicas individuais (Jonas, Rodrigues e Resck, 2011) e na execução de atividades de educação em saúde (Roecker, Budó e Marcon, 2012). Experiências internacionais debatidas por Giovanella (2014) demonstram que o enfermeiro da APS, ao assumir funções clínicas, pode ofertar atenção em saúde de qualidade similar à prestada pelo médico, além de ser capacitado para o exercício de funções de coordenação com gestão clínica e de casos, gerenciando o cuidado na rede.

A quase totalidade das EqABs informou que realizava atendimentos de urgência/emergência, percentual expressivamente maior que a média 
nacional para municípios de grande porte (Fausto et al., 2014). O alto percentual de usuários que procuravam o atendimento na própria UBS para casos de urgência/emergência indicou o reconhecimento de que a unidade era um recurso e também uma porta de entrada para esses casos. Entretanto, complementarmente, é importante entender quais foram os motivos que deixaram sem atendimento um quinto dos que procuraram sua UBS.

A oferta de procedimentos/pequenas cirurgias pelas EqABs também foi superior à média nacional (Fausto et al., 2014), o que caracterizou um modelo mais resolutivo. $\mathrm{O}$ fato de usuários de serviços de $\mathrm{AB}$ referirem que as equipes buscavam resolver seus problemas sugeriu que eles estavam satisfeitos com a atenção, o que é um forte preditor da manutenção daquela unidade ou equipe como fonte regular de cuidado (Starfield, 2002).

Entretanto, o acesso aos medicamentos prescritos apareceu como um entrave à resolutividade, embora o município apresentasse desempenho superior aos demais com população acima de quinhentos mil habitantes (Fausto et al., 2014). Essa informação pode indicar problemas de abastecimento ou a inclusão de prescrições fora da 'Relação Nacional de Medicamentos Essenciais'.

$\mathrm{O}$ acesso aos resultados dos exames foi uma questão que merece ser avaliada na organização do processo de trabalho. Como se mostrou alto o percentual dos que informaram dificuldades, as consequências podem incluir tensionamentos desnecessários entre usuários e equipe, bem como o aumento da busca de consultas médicas sem necessidade.

O apoio matricial como estratégia para aumentar a resolutividade, a qualidade e a continuidade do cuidado parecia estar institucionalizado pela gestão municipal e fazia parte do cotidiano das equipes. De acordo com os resultados, havia troca sistemática de conhecimentos entre diferentes especialidades e as EqABs. Entre os especialistas, o psicólogo era o mais atuante, e menor a participação das especialidades médicas, com exceção do psiquiatra, que figurava entre os quatro especialistas que mais apoiavam as equipes.

$\mathrm{O}$ atendimento do usuário pelo mesmo profissional era mais comum no caso do médico do que no do enfermeiro. Entretanto, o instrumento não permitiu identificar com clareza os motivos - se correspondiam a uma lógica de organização do processo de trabalho nas unidades com várias equipes ou se havia, de fato, rotatividade na composição delas, um aspecto importante a se aprofundar em outros estudos.

Ainda que tenha sido alto o percentual de usuários que referiram ser atendidos pelos mesmos profissionais, foi preocupante que um terço tenha afirmado que eles nunca se lembravam do que acontecera nas consultas anteriores. Para Starfield (2002), os usuários entendiam melhor os conselhos e tinham maior probabilidade de aderir a um plano terapêutico se o profissional demonstrasse interesse e apresentasse informações no início da consulta. 
O município alcançou cobertura quase total de prontuários eletrônicos na $\mathrm{AB}$, o que sem dúvida favorecia a coordenação horizontal. Contudo, foi incipiente a utilização de tecnologias de informação e comunicação, como o Telessaúde, que permitiria, por exemplo, consultas ou discussão de casos com especialistas, sem necessidade de deslocamento do usuário. Esse tipo de prática tem potencial para abreviar o tempo de espera para consultas especializadas, reduzir custos e melhorar a coordenação do cuidado (Bodenheimer, 2008).

Para a marcação de consultas, o telefone foi um instrumento pouco utilizado, em concordância com o achado de Fausto e colaboradores (2014) para o país. Em contrapartida, observou-se que o ACS foi o principal intermediário para marcação de consultas, tanto na $\mathrm{AB}$ quanto na comunicação do agendamento de procedimentos/consultas especializadas.

A coordenação do cuidado é particularmente desafiadora em sistemas nos quais os pontos de atenção atuam de forma fragmentada. Conforme outros estudos (Almeida et al., 2010), ainda é fraca a coordenação entre APS e atenção hospitalar, como aponta a percepção dos profissionais quanto à disponibilidade de centrais de regulação de leitos, pelo menos para os casos de cirurgias eletivas. Em relação à atenção especializada, parte dos usuários ainda busca atendimento sem a marcação garantida, ou seja, de forma individualizada e sem garantia do acesso.

Outra barreira à coordenação foi a baixa disponibilidade de prontuários eletrônicos compartilhados. Uma vez que o recebimento da contrarreferência por meio das tradicionais guias em papel era débil e que mais da metade das EqABs não conversavam sobre o atendimento recebido fora da UBS, cabe indagar sobre as possibilidades de continuidade do cuidado, grau em que os usuários experimentam a coordenação (Haggerty, Yavich e Báscolo, 2009).

$\mathrm{O}$ estabelecimento de acordos entre profissionais da APS e especialistas de referência, com definição de responsabilidades, constitui-se importante medida pró-coordenação (Forrest et al., 2000; Bodenheimer, 2008). Forrest e colaboradores (2000) concluíram que a referência entre médicos da APS e especialistas aumenta a satisfação dos primeiros com a atenção especializada e melhoram os resultados obtidos com o encaminhamento. Os resultados do PMAQ-AB demonstraram que parece ser prática pouco frequente o contato entre profissionais das EqABs e demais especialistas e ainda menos habitual o processo inverso.

Por fim, conforme apontado por Bodenheimer (2008), além das inovações que podem contribuir para melhor coordenação, sistemas de saúde com forte base de APS apresentam maiores possibilidades de enfrentamento da fragmentação do cuidado. Nesse sentido, ainda que de forma tardia, o município do Rio de Janeiro define em sua política de saúde que a AB deve constituir-se no centro da RAS, experimentando uma rápida expansão. $\mathrm{O}$ modelo adotado - as clínicas da família -, grandes unidades básicas com 
cinco ou mais equipes, parecem favorecer o acesso aos cuidados em saúde, tanto na disponibilidade ou abrangência das ações quanto no acolhimento (Alves et al., 2014). Os resultados do PMAQ-AB parecem indicar que a $A B$ se consolidava, no município, como porta de entrada preferencial, na medida em que os usuários identificaram que as EqABs buscavam resolver seus problemas de saúde e que as UBSs eram dotadas de insumos básicos para realização de pequenos procedimentos. Além disso, o prontuário eletrônico estava disponível para a $\mathrm{AB}$, os profissionais recebiam apoio matricial de forma frequente e as reuniões semanais pareciam institucionalizadas.

Ainda assim, cabem estratégias para enfrentamento dos tempos de espera na $\mathrm{AB}$, fornecimento de medicamentos básicos, acesso aos resultados de exames e discussões sobre as competências profissionais dos diversos atores envolvidos no cuidado, de forma a superar a burocratização e a verticalização das relações com os usuários. Nessa direção, Franco (2009) alerta que apenas a ampliação da ESF não é suficiente para a transposição do lócus de trabalho do ambulatório para o domicílio ou do hospital para a UBS, quando os processos de trabalho permanecem inalterados ou o núcleo tecnológico conserva-se centrado no procedimento/doença e não no usuário.

Em relação à integração com a RAS, os tempos de espera para atendimento especializado ainda eram altos e a comunicação entre os profissionais realizada predominantemente pelas guias de referência, com baixa utilização de TICs, inclusive para as ações de apoio matricial. Ressalta-se que a AB não conforma rede de forma isolada, pela simples extensão de cobertura. Ao mesmo tempo, não há rede sem ampla cobertura de $\mathrm{AB}$, sem capacidade instalada e gestão do cuidado planejada de forma sistêmica.

Em síntese, ao se considerarem as duas macroestratégias para ampliação das possibilidades de coordenação do cuidado pela $\mathrm{AB}$, os resultados pareceram indicar que, no município, foram identificados avanços mais expressivos no fortalecimento das dimensões essenciais da $\mathrm{AB}$ e localizados desafios importantes para a integração da Rede de Atenção à Saúde, que, de certo modo, minimizavam as possibilidades de coordenação do cuidado pelas EqABs. Não obstante, a pesquisa apresentou alguns limites, posto que analisou dados frios dos números que emergiram da avaliação do PMAQ-AB, ou seja: reconhece-se a necessidade de abordagens complementares que incorporem a perspectiva do usuário por meio de estudos de outra natureza, possibilitando, assim, com base na triangulação de métodos, comparações entre estudos e desvelamento do 'não dito'. 


\section{Colaboradoras}

Patty Fidelis de Almeida, Juliana Marin e Elisete Casotti foram responsáveis pela concepção, delineamento e redação do artigo. Patty Fidelis de Almeida fez a revisão crítica do manuscrito. Não há conflito de interesses.

Resumen El estudio presentado aquí estudió la coordinación del cuidado por medio de datos del Programa Nacional para la Mejora de la Calidad y del Acceso de la Atención Básica. Se trató de un estudio descriptivo en base a cuestionarios aplicados a 1.313 usuarios y 324 equipos de atención básica en el municipio de Río de Janeiro (Brasil) en 2012. Se evaluaron dimensiones como organización de la puerta de entregada, resolutividad y continuidad del cuidado, integración horizontal, organización de los flujos y acceso a la red de referencia, continuidad informativa y comunicación entre profesionales. Los resultados indicaron que la atención primaria en salud se consolidó como puerta de entrada preferente. Los usuarios manifestaron que los equipos de atención básica buscaban resolver sus problemas de salud, el prontuario electrónico estaba disponible, aunque estuviera integrado a los demás niveles, y los profesionales indicaron realizar reuniones semanales y recibir apoyo matricial. Sin embargo, el tiempo de espera para atención especializada era alto y la comunicación entre los profesionales insuficiente, lo que dificultaba el recorrido del usuario en la búsqueda de cuidado y revelaba las fragilidades del trabajo en red. Se identificaron avances en el fortalecimiento de la atención primaria y desafíos para la constitución de la Red de Atención de la Salud que minimizaban las posibilidades de coordinación del cuidado por parte de los equipos de atención básica.

Palabras clave evaluación de servicios de salud; atención primaria de la salud; estrategia salud de la familia; coordinación del cuidado.

\section{Notas}

1 Universidade Federal Fluminense, Departamento de Planejamento em Saúde, Instituto de Saúde Coletiva, Niterói, Rio de Janeiro, RJ.

<pattyfidelis@id.uff.br>

Correspondência: Rua Marquês do Paraná, 303, $3^{\circ}$ andar, CEP 24030-2100, prédio anexo ao Hospital Universitário Antonio Pedro, Centro, Niterói, Rio de Janeiro, Brasil.

2 Programa de Pós-Graduação em Bioética, Ética Aplicada e Saúde Coletiva (PPGBIOS), por associação entre a Universidade Federal Fluminense, Universidade Federal do Rio de Janeiro, Fundação Oswaldo Cruz e Universidade do Estado do Rio de Janeiro, Niterói, Rio de Janeiro, Brasil.

<jubsmarin@yahoo.com.br>

3 Universidade Federal Fluminense, Departamento de Planejamento em Saúde, Instituto de Saúde Coletiva, Niterói, Rio de Janeiro, Brasil.

$<$ elisete.casotti@gmail.com> 
4 Citadas no texto como equipes de atenção básica (EqABs), em concordância com a nomenclatura adotada pelo PMAQ-AB.

\section{Referências}

ALMEIDA, Patty F. et al. Desafios à coordenação dos cuidados em saúde: estratégias de integração entre níveis assistenciais em grandes centros urbanos. Cadernos de Saúde Pública, Rio de Janeiro, v. 26, n. 2, p. 286$-298,2010$.

ALVES, Márcia G. M. et al. Fatores condicionantes para o acesso às equipes da Estratégia Saúde da Família no Brasil. Saúde em Debate, Rio de Janeiro, v. 38, n. especial, p. 34-51, 2014.

BODENHEIMER, Thomas. Coordinating care: a perilous journey through the Health Care System. The New England Journal of Medicine, Whaltam, v. 358, n. 10, p. 1.064-1.071, 2008.

BOERMA, Wienke G. W. Coordenação e integração em atenção primária europeia. In: SALTMAN, Richard B.; RICO, Ana; BOERMA, Wienke G. W. (orgs.). Atenção primária conduzindo as redes de atenção à saúde: reforma organizacional na atenção primária europeia. Berkshire: Open University Press, 2010. p. 25-47.

BORNSTEIN, Vera J.; DAVID, Helena M. S. L. Contribuições da formação técnica do agente comunitário de saúde para o desenvolvimento do trabalho da equipe Saúde da Família. Trabalho, Educação e Saúde, Rio de Janeiro, v. 12, n. 1, p. 107-128, abr. 2014.

BRASIL. Ministério da Saúde. Secretaria de Atenção à Saúde. Departamento de Atenção Básica. Avaliação para melhoria da qualidade da equipe de saúde da família. Brasília, DF: Ministério da Saúde, 2005.
BRASIL. Ministério da Saúde. Secretaria de Atenção à Saúde. Departamento de Atenção Básica. Programa Nacional de Melhoria do Acesso e da Qualidade da Atenção Básica. Saúde mais perto de você: acesso e qualidade da atenção básica (PMAQ-AB). Brasília, DF: Ministério da Saúde, 2012a.

BRASIL. Ministério da Saúde. Secretaria de Atenção à Saúde. Departamento de Atenção Básica. Política Nacional de Atenção Básica. Portaria 2.488, de 21 de outubro de 2011. Série E. Legislação em Saúde. Brasília, DF: Ministério da Saúde, 2012b.

BRASIL. Ministério da Saúde. Departamento de Atenção Básica. Histórico de cobertura da saúde da família. 2015a. Disponível em: $<$ http://dab.saude.gov.br/portaldab/historico_ cobertura_sf.php > . Acesso em: 5 nov. 2015.

BRASIL. Ministério da Saúde. Departamento de Atenção Básica. Programa de Melhoria do Acesso e da Qualidade. 2015b. Disponível em: $<$ http://dab.saude.gov.br/portaldab/ape_pmaq. php?conteudo=1_ciclo $>$. Acesso em: 10 nov. 2015.

BYNUM, Julie P. W.; ROSS, Joseph S. A measure of care coordination? Journal of General Internal Medicine, v. 28, n. 3, p. 336-338, 2012.

CARNEIRO, Carla C. G.; MARTINS, Maria I. C. Novos modelos de gestão do trabalho no setor público de saúde e o trabalho do agente comunitário de saúde. Trabalho, Educação e Saúde, Rio de Janeiro, v. 13, n. 1, p. 45-66, abr. 2015.

FAUSTO, Márcia C. R. et al. A posição da Estratégia Saúde da Família na Rede de Atenção 
à Saúde na perspectiva das equipes e usuários participantes do PMAQ-AB. Saúde em Debate, Rio de Janeiro, v. 38, n. especial, p. 34-52, 2014.

FORREST, Christopher B. et al. Coordination of specialty referrals and physician satisfaction with referral care. Archives of Pediatrics and Adolescent Medicine, Michigan, v. 154, n. 5, p. 499-506, 2000.

FRANCO, Túlio B. Transição tecnológica e inclusão social na saúde. In: FRANCO, Túlio, B.; ANDRADE, Cristina S.; FERREIRA, Vitória S. C. (orgs.). A produção subjetiva do cuidado: cartografias da Estratégia Saúde da Família. São Paulo: Hucitec, 2009. p. 147-162.

GABRIEL, Juliana O; CASOTTI, Elisete. Organização da atenção em saúde bucal na cidade do Rio de Janeiro: resultados do Programa de Melhoria do Acesso e Qualidade da Atenção Básica. Revista de APS, Juiz de Fora, v. 19, n. 2, p. 245-260, 2016.

GIOVANELLA, Lígia. Atenção primária à saúde e coordenação dos cuidados na rede assistencial. Divulgação em Saúde para Debate, Rio de Janeiro, n. 51, p. 30-37, 2014.

HAGGERTY, Jeannie L.; YAVICH, Natalia; BÁSCOLO, Ernesto P. Grupo de Consenso sobre un Marco de Evaluación de la Atención Primaria en América Latina: un marco de evaluación de la atención primaria de salud en América Latina. Revista Panamericana de Salud Pública, Washington DC, v. 26, n. 5, p. 377-384, 2009.

HARZHEIM, Erno (org.). Reforma da atenção primária à saúde na cidade do Rio de Janeiro: avaliação dos três anos de clínicas da família. Pesquisa avaliativa sobre aspectos de implantação, estrutura, processo e resultados das clínicas da família na cidade do Rio de Janeiro. Porto Alegre: Organização Pan-Americana da Saúde, 2013. (Série Técnica Inovação na Gestão).

JONAS, Lucélia T.; RODRIGUES, Hugo C.; RESCK, Zélia M. R. A função gerencial do enfermeiro na Estratégia Saúde da Família: limites e possibilidades. Revista de APS, v. 14, n. 1, p. 28-38, 2011.

KRINGOS, Dionne S. et al. The breadth of primary care: a systematic literature review of its core dimensions. BMC Health Services Research, London, v. 10, n. 1, p. 65-78, 2010.

LIMA, Danielle M. C. Estratégia Saúde da Família na cidade do RJ: desafios da atenção primária numa grande cidade. $157 \mathrm{f}$. Dissertação (Mestrado) - Escola Nacional de Saúde Pública Sergio Arouca, Fundação Oswaldo Cruz, Rio de Janeiro, 2014.

McDONALD, Kathryn M. et al. Care coordination measures atlas. Rockville, MD: Agency for Healthcare Research and Quality, 2011. Disponível em: <www.ahrq.gov/qual/ careatlas.pdf $>$. Acesso em: 17 out. 2012.

NOLTE, Ellen; McKEE, Martin. Integration and chronic care: a review. In: NOLTE, Ellen; McKEE, Martin (eds.). Caring for people with chronic conditions: a health system perspective. Brussels: World Health Organization on Behalf of the European Observatory on Health Systems and Policies, 2008. p. 64-91.

ROECKER, Simone; BUDÓ, Maria L. D.; MARCON, Sonia S. Trabalho educativo do enfermeiro na Estratégia Saúde da Família: dificuldades e perspectivas de mudanças. Revista da Escola de Enfermagem da USP, São Paulo, v. 46, n. 3, p. 641-649, 2012.

SCHANG, Laura; WAIBEL, Sina; THOMSON, Sarah. Measuring care coordination: health system and patient perspectives. Report prepared for the Main Association of Austrian Social Security Institutions. London: LSE Health, 2013.

RIO DE JANEIRO. Secretaria Municipal de Saúde e Defesa Civil (SMSDC). Superintendência de Atenção Primária. Guia de Referência Rápida. Carteira de serviços: relação de serviços prestados na atenção primária à saúde. Rio de Janeiro: SMSDC, 2011.

SORANZ, Daniel. Reforma da atenção primária: panorama atual da atenção primária 
no Rio de Janeiro. Rio de Janeiro, 2012. Apresentado no VIII Ciclo de Debates Conversando sobre a Estratégia Saúde da Família, Escola Nacional de Saúde Pública Sergio Arouca, Fundação Oswaldo. Disponível em: $<$ www5.ensp.fiocruz.br/biblioteca/dados/ txt_782042831.ppt>. Acesso em: 9 maio 2015.

STARFIELD, Barbara. Atenção primária: equilíbrio entre necessidades de saúde, serviços e tecnologia. Brasília, DF: Unesco, Ministério da Saúde, 2002.
VÁZQUEZ, Maria L. et al. Organizaciones sanitarias integradas: un guía para el análisis. Revista Española de Salud Pública, Madrid, v. 79, n. 6, p. 633-643, 2005.

Recebido em 10/08/2015

Aprovado em 29/03/2016 